HÕ DİNH DUÂ'N

KODAI MATH. J.

17 (1994), 390-394

\title{
A NOTE ON GORENSTEIN DIMENSION AND THE AUSLANDER-BUCHSBAUM FORMULA
}

\author{
BY Hõ Dình DuÂ'N
}

\section{Introduction}

In [1] a generalization of the classical Auslander-Buchsbaum formula is made. Namely, if $R$ is a noetherian local commutative ring and $M$ is an $R$-module of finite Gorenstein dimension, then one has

$$
G-\operatorname{dim} M+\operatorname{depth} M=\operatorname{depth} R
$$

where $G$-dim $M$ denotes the Gorenstein dimension of the module $M$. It is worth noting an important fact in this setting: If $M$ has finite Gorenstein dimension then

$$
G-\operatorname{dim} M=\sup \left\{t ; \operatorname{Ext}_{R}^{t}(M, R) \neq 0\right\}
$$

This equality leads us to define the so called weak Gorenstern dimension. Let $R$ be a commutative noetherian ring. Hereafter the notation $\operatorname{Ext}_{R}^{t}(M, R)$ will be abbreviated to $\operatorname{Ext}^{t}(M, R)$, unless otherwise specified.

Definition. We say that a finitely generated $R$-module $M$ has weak Gorenstein 0 if $\operatorname{Ext}^{t}(M, R)=0$ for all $t>0$. If $k>0$, we say that $M$ has weal Gorenstein dimension (written w.g.d $(M)=k$ ) if $\operatorname{Ext}^{t}(M, R)=0$ for all $t>k$ while $\operatorname{Ext}^{k}(M, R) \neq 0$. Also we put w.g.d $(M)=\infty$ if $\operatorname{Ext}^{t}(M, R) \neq k$ for all $k=0,1,2, \ldots$

From this definition we see that every projective module $P$ has w.g.d. $(P)=0$, and it turns out that the class of all zero-dimensional modules plays an important role in our stúdy so we also give

Definition. We denote by $\mathcal{C}_{0}\left(=\mathcal{C}_{0}(R)\right)$ the family of finitely generated $R$-modules $M$ for which w.g.d $(M)=0$.

Let $R$ be a local ring, i.e. there is a unique maximal ideal $\mathcal{M}$. The depth of a finitely generated $R$-module $M$ can be defined by the formula

$$
\operatorname{depth}(M)=\inf \left\{k: \operatorname{Ext}^{k}(R / \mathfrak{M}, M) \neq 0\right\} .
$$

Now we announce the main results in this note.

THEOREM A. Let $R$ be a local noetherian ring. Assume that every module in the class is reflexive. Then

$$
\text { w.g.d }(M)+\operatorname{depth}(M)=\operatorname{depth}(R)
$$

Received May 30, 1993. 
for every finitely generated $R$-moduel $M$ of finete weak Gorenstern dimension.

Let $R$ be a noetherian ring. Recall that if $M$ is a non-zero and finitely generated $R$-module then its grade number is the smallest integer $\mathrm{k}$ such that $\operatorname{Ext}^{t}(M, R) \neq 0$. We say that $M$ is weakly perfect if

$$
\operatorname{grade}(M)=\text { w.g.d }(M)
$$

TheOREM B. Let $R$ be as in Theorem $A$. We assume furhtermore that $R$ is CohenMacaulay. Then a finitely generated $R$-module $M$ is weakly perfect if and only if $M$ is a Cohen-Macaulay moduel of finıte weak Gorenstern dimension.

THEOREM C. Let $R$ be a commutative noetherian ring (not necessarily local) such that every localization of $R$ at a prime ideal satisfies the hypothesis of Theorem $A$. Let $M$ be a finitely generated $R$-module. If w.g.d $\left(M_{\mathfrak{p}}\right)<\infty$ for every prime ideal $\mathfrak{p}$ then

$$
\operatorname{grade}\left(\operatorname{Ext}^{t}(M, R)\right) \geq t
$$

for each $t=0,1,2, \ldots$ Consequently, if $R$ is local and satısfies the hypothesis of Theorem $A$, then $R$ is Gorenstern if and only if every finitely generated $R$-module has finite weak Gorenstein dimension.

Remark. Concerning the condition 'all modules in $\mathcal{C}_{0}(R)$ are reflexive', some comments are worth noting here. First we see that all Gorenstein rings satisfy the hypothesis of Theorem A. This follows from the bidualizing formula in such rings, see e.g. Fossum [4]. Also, there are non-Gorenstein local rings for which this hypothesis holds. For this we take a local artin ring $(R, \mathfrak{M})$ with $\mathfrak{M}^{2}=0$, then in this ring all finitely generated modules of weak Gorenstein dimension 0 are reflexive (in fact they are even free, see Menzin [6]). This has been generalized to the case $\mathfrak{M}^{3}=0$ in a remark made by J-E Roos and was proved by T. Ekedahl. We refer to the Examples at the end of this note for more details.

Then it reveals as a task to find a local artin ring $R$ and a module $M$ in $C_{0}(R)$ such that $M$ is reflexive and $M^{*}$ is not in $C_{0}(R)$. The proofs of the three theorems below occur in Section 2. They more or less rely on the relating materials in Auslander-Bridger [1] and Bruns-Herzog [3], especially many techniques used here are similar to those in [1]. First we need some preliminaries in Section 1.

I wish to thank E.K. Ekstrom, J-E Bjork, J-E Roos, T. Ekedahl and R. Froberg for their stimulating discussions.

\section{Some preliminaries on weak Gorenstein dimension and depth.}

We list a series of preliminary results mostly concerning the case when every zelc divisor of the ring is a unit. Below $R$ is a local noetherian ring such that every module in $C_{0}(R)$ is reflexive and $M$ denotes a finitely generated $R$-module.

(1) If $\operatorname{depth}(R)=0$ and $M^{*}=0$, then $M=0$.

(2) If $\operatorname{depth}(R)=0$ and $0 \neq M=M^{* *}$, i.e. $M$ is reflexive, then $\operatorname{depth}(M)=0$. 
(3) If $0 \rightarrow K \rightarrow X \rightarrow M \rightarrow 0$ is an exact sequence where $X \in \mathcal{C}_{0}$ and $0<$ w.g.d( $\left.M\right)<$ $\infty$, then

$$
\text { w.g.d }(K)=\text { w.g.d }(M)-1
$$

(4) If $\operatorname{depth}(R)=0$ and w.g.d $(M)<\infty$ then w.g.d $(M)=0$.

(5) Let $\mathfrak{M}$ be the maximal ideal of the local ring $R$ and $x \in \mathfrak{M}$ be $R$-regular. Then the quotient ring $R / x R$ also satisfy the hypothesis of Theorem $\mathrm{A}$, i.e. all modules in $\mathcal{C}_{0}(R / x R)$ are reflexive.

Above (1) and (3) are obvious (for those we do not need the hypothesis of Theorem A). (2) follows since $M=M^{* *}$ contains the non-zero submodule $\operatorname{Hom}_{R}\left(M^{*}, R / \mathfrak{M}\right)$ which is annihilated by $\mathfrak{M}$. There remains to prove (4) and (5).

We proceed by an induction over $k$. Let $k \geq 1$ and assume that if w.g.d $(M)=k$, then w.g.d $(M)=0$. Then, if w.g.d $(M)=k+1$ we apply (3) and the induction hypothesis on $k$ to get w.g.d $(M) \leq 1$. Hence there only remains to prove that when w.g.d $(M)=1$, it follows that w.g.d $(M)=0$.

To prove this we take an exact sequence

$$
0 \rightarrow K \rightarrow X \rightarrow M \rightarrow 0
$$

Where $X$ is a free $R$-module of finite rank. Here w.g.d $(K)=0$ and hence $K=K^{* *}$ by the hypothesis. The dualizing sequence gives:

$$
0 \rightarrow M^{*} \rightarrow X^{*} \rightarrow K^{*} \rightarrow \operatorname{Ext}^{1}(M, R) \rightarrow 0
$$

Let $N=\operatorname{Im}(X * \rightarrow K *)$. This gives the two exact sequences

$$
\begin{aligned}
& 0 \rightarrow \operatorname{Ext}^{1}(M, R)^{*} \rightarrow K \rightarrow N^{*} \rightarrow \operatorname{Ext}^{1}\left(\operatorname{Ext}^{1}(M, R), R\right) \rightarrow 0 \\
& 0 \rightarrow N^{*} \rightarrow X \rightarrow M^{* *} \rightarrow \operatorname{Ext}^{1}(N, R) .
\end{aligned}
$$

Since $K \rightarrow X$ is injective we conclude that $K \rightarrow N^{*}$ is injective in (i) and hence $\operatorname{Ext}^{1}(M, R)^{*}=0$. Then (1) gives $\operatorname{Ext}^{1}(M, R)=0$ which proves that w.g.d $(M)=0$. Finally (5) follows from the following observation: for any $R / x R$-module $M$ and any integer $t$ we have an isomorphism of $R / x R$-modules

$$
\operatorname{Ext}_{R}^{t}(M, R) \cong \operatorname{Ext}_{R / x R}^{t-1}(M, R / x R)
$$

\section{Proof of Theorem A}

We proceed by an induction on $n-\operatorname{depth}(R)$.

Assume first that $n=0$. If w.g.d $(M)<\infty$, one has $M \in \mathcal{C}_{0}$ by (4) and then $M$ is reflexive by the hypothesis. Hence $\operatorname{depth}(M)=0$ by $(2)$ and the required formula in Theorem 1 holds.

Let $n \geq 1$ and assume that Theorem A holds for local rings of depth $\leq n-1$. Let $M$ be some $R$-module with w.g.d $(M)<\infty$. Suppose also that $\operatorname{depth}(M)>0$. Let $\mathfrak{M}$ be the maximal ideal of $R$. Then there exists some $x \in \mathfrak{M}$ such that $x$ is a non-zero divisor on both $R$ and $M$. It follows that

(1) $\operatorname{depth}(M / x M)=\operatorname{depth}(M)-1$

(2) $\operatorname{depth}(R / x R)=\operatorname{depth}(R)-1$.

Moreover by standard homological algebra one has:

$$
\text { w.g. } \mathrm{d}_{R / x R}(M / x M)=\text { w.g. } \mathrm{d}_{R}(M)
$$


The induction hypothesis applies to the ring $R / x R$ (by (5)) and the module $M / x M$. We conclude that the formula in Theorem A holds for the $R$-module $M$.

To finish the induction over $n$ there remains to consider the case when $\operatorname{depth}(M)=0$. In this case we consider an exact sequence

$$
0 \rightarrow K \rightarrow X \rightarrow M \rightarrow 0
$$

where $X$ is a free $R$-module of finite rank. Since $\operatorname{depth}(R)>0$ we get the long exact sequence

$$
\cdots \rightarrow \operatorname{Ext}^{0}(R / \mathfrak{M}) \rightarrow \operatorname{Ext}^{1}(R / \mathfrak{M}, K) \rightarrow \operatorname{Ext}^{1}(R / \mathfrak{M}, X) \rightarrow \operatorname{Ext}^{1}(R / \mathfrak{M}, M) \rightarrow \cdots
$$

Here $\operatorname{Ext}^{0}(R / \mathfrak{M}, M) \neq 0$ since $\operatorname{depth}(M)=0$ and hence

$$
\operatorname{Ext}^{1}(R / \mathfrak{M}, K)=0
$$

This implies that depth $(K)=1$. Moreover (3) implies that $K$ has finite weak Gorenstein dimension and then the first part of the proof gives

$$
\text { w.g.d }(K)=\operatorname{depth}(R)-1 \text {. }
$$

Finally we notice that $w g d(M)$ must be positive for if w.g.d $(M)=0$ the hypothesis implies that $M$ is a submodule of $A$ free $R$-module and then its depth would be positive. When w.g.d $(M)>0$ we see that (i) and (3) give w.g.d $(M)=\operatorname{depth}(R)$ which gives the required formula in Theorem $\mathrm{A}$ and finishes the induction over $n$.

Proof of Theorem B. Since $R$ is Cohen-Macaulay one has

$$
\operatorname{depth}(R)=\operatorname{dim}(R)=\operatorname{dim}(M)+\operatorname{grade}(M)
$$

for every $R$-module $M$. If w.g.d $(M)<\infty$ one has

$$
\operatorname{depth}(M)+\text { w.g.d }(M)=\operatorname{depth}(R)
$$

by Theorem $\mathrm{A}$. We conclude that $M$ is weakly perfect if and only if $\operatorname{dim}(M)=\operatorname{depth}(M)$. Since the last equality olds if and only if $M$ is Cohen-Macaulay we get Theorem B. (Note: for the implication ' $M$ weakly perfect $\Longrightarrow M$ Cohen-Macaulay of finite weak Gorenstein dimension' we do not need the hypothesis that $R$ is local.)

Proof of Theorem $C$. Recall that for any $R$-module $N$,

$$
\operatorname{grade}(N)=\inf \left\{\operatorname{depth}\left(R_{\mathfrak{p}}\right): \mathfrak{p} \in \operatorname{Supp}(N)\right\}
$$

Let $\mathfrak{p} \in \operatorname{Supp}\left(\operatorname{Ext}^{t}(M, R)\right)$, then $\operatorname{Ext}_{\mathfrak{p}}^{t}\left(M_{\mathfrak{p}}, R_{\mathfrak{p}}\right) \cong \operatorname{Ext}_{R}^{t}(M, R)_{\mathfrak{p}} \neq 0$. This implies w.g.d $\left(M_{\mathfrak{p}}\right) \geq t$ by the definition of the weak Gorenstein dimension. Now since w.g.d $\left(M_{\mathfrak{p}}\right)$ $<\infty$ Theorem A gives

$$
\operatorname{depth}\left(R_{\mathfrak{p}}\right)=\text { w.g.d }\left(M_{\mathfrak{p}}\right)+\operatorname{depth}\left(M_{\mathfrak{p}}\right) \geq t,
$$

and the first part of Theorem $C$ is proved. For the second assertion we have only to prove the "if" part since every finitely generated $R$-module then already has finite Gorenstein dimension. To prove this we show that $\operatorname{inj} \cdot \operatorname{dim}(M)$ is not greater than the depth of the ring which is finite since $R$ is local, and this is a consequence of the first part we have just shown.

Examples. (1) Let $(R, \mathfrak{M})$, be a local artin ring with $\mathfrak{M}^{2}=0$ and which is not Gorenstein (i.e. when the length of $\mathfrak{M} \geq 2$.) Then any finitely generated $R$-module $M$ with w.g.d $(M)=0$ is free, and hence reflexive. 
Proof. This is a consequence of [6, Proposition 2].

(2) Let $(R, \mathfrak{M})$ be a local Cohen-Macaulay ring which is not Gorenstein. If some s.o.p. is not in $\mathfrak{M}^{2}$ and $R / \mathfrak{M}^{2}$ is artin, then we have the same conclusion as in (1).

Proof. This is a consequence of [6, Proposition 7].

(3) (Ekedahl) Let $(R, \mathfrak{M})$ be a local artin ring the $\mathfrak{M}^{3}=0$. Then every module whose weak Gorenstein dimension is 0 , is free. This remark is given by T.Ekedalh of Stockholm in a private communication with the author.

\section{REFERENCE}

[1] M. Auslander and M. Bridger, Stable module theory, Mem. Amer. Math. Soc. 94 (1969).

[2] R. Bogvad, Gorenstein rings with transcendental Poincare-series, Math. Scand. 53 (1983), 5-15.

[3] W. Bruns and J. Herzog, Cohen-Macaulay rings, in preparation, 1992.

[4] T. Ekedahl, Private communication.

[5] R. Fossum, Duality over Gorenstein rings, Math. Scand. 26 (1970), 165-176.

[6] H. Matsumura, Commutative ring theory, Cambridge Univ. Press, 1986.

[7] M.S. Menzin, The Condition $\operatorname{Ext}(M, R)=0$ for modules over local artin algebras $(R, \mathfrak{M})$ with $\mathfrak{M}^{2}=0$, Proc. Amer. Math. Soc. 43 (1971), 47-52.

[8] J-F Roos, Finiteness conditions in commutative algebra and solution of a problem of Vasconcelos, London Math. Soc. Lect. notes, Cambridge Univ. Press, 1983.

Department of Mathematics

UNIVERSITY OF HUẼ DạI HọC

Tô'NG Họp HUẼ, ViệtNam

Present AdDREss:

COMPuTER SCIENCE Division

Asian InStitute of TeCHNOLOGY

BOX 2754, BANGKOK 10501

Thailand

e-mail: duan@cs.ait.ac.th 